\title{
Fondaparinux Sodium
}

National Cancer Institute

\section{Source}

National Cancer Institute. Fondaparinux Sodium. NCI Thesaurus. Code C47539.

The sodium salt form of fondaparinux, a synthetic glucopyranoside with antithrombotic activity. Fondaparinux sodium selectively binds to antithrombin III, thereby potentiating the innate neutralization of activated factor X (Factor Xa) by antithrombin. Neutralization of Factor Xa inhibits its activity and interrupts the blood coagulation cascade, thereby preventing thrombin formation and thrombus development. (NCI05) 